Revue des patrimoines

43 | 2021

Des écoles d'art académiques aux écoles d'art : des collections et des lieux, un patrimoine à valoriser

\title{
L'influence de Paris et de Rome dans la constitution de collections académiques en Europe
}

Des collections de plâtres gréco-romains aux modèles nationaux

Paris and Rome's influence on the constitution of academic collections in Europe.

From the Greco-Roman plaster casts collections to the national models

Morwena Joly-Parvex

\section{(2) OpenEdition}

1 Journals

Édition électronique

URL : http://journals.openedition.org/insitu/28407

DOI : 10.4000/insitu.28407

ISSN : 1630-7305

Éditeur

Ministère de la Culture

Référence électronique

Morwena Joly-Parvex, «L'influence de Paris et de Rome dans la constitution de collections

académiques en Europe », In Situ [En ligne], 43 | 2021, document 2, mis en ligne le 12 janvier 2021

consulté le 14 janvier 2021. URL : http://journals.openedition.org/insitu/28407 ; DOI : https://doi.org/ $10.4000 /$ insitu. 28407

Ce document a été généré automatiquement le 14 janvier 2021.

\section{(c) $(1) \Theta \Theta$}

In Situ Revues des patrimoines est mis à disposition selon les termes de la licence Creative Commons Attribution - Pas d'Utilisation Commerciale - Pas de Modification 4.0 International. 


\section{L'influence de Paris et de Rome dans la constitution de collections académiques en Europe}

Des collections de plâtres gréco-romains aux modèles nationaux

Paris and Rome's influence on the constitution of academic collections in Europe. From the Greco-Roman plaster casts collections to the national models

Morwena Joly-Parvex

1 Les principales académies européennes, ainsi que les écoles d'art qui se multiplient sur l'ensemble des territoires, soutenues par les capitales de leur pays, ont été fondées tout au long $\mathrm{du}$ XVIII ${ }^{\mathrm{e}}$ siècle, avec pour références principales les Académies de Paris et de Rome, tant sur le plan de leur fonctionnement que de leur enseignement. Le séjour à Paris et à Rome s'affirme comme un incontournable prolongement à la formation artistique, au regard de leur vitalité artistique. La France s'est par ailleurs implantée à Rome, sous l'espèce de l'Académie de France fondée sous Louis XIV en 1666, et offre aux visiteurs qui le souhaitent une collection de plâtres antiques qui fascine les académies européennes. Il s'agit de valoriser un "grand genre ", digne d'une nation moderne, projet qui se trouve au fondement de toute fondation académique d'art dans les capitales européennes.

2 À la faveur de la circulation intense des personnes et des œuvres à la fin du XviII siècle et dans la continuité de la culture humaniste de la Renaissance, la célébration européenne de l'antique s'intensifie, fruit du cosmopolitisme des Lumières. La référence antique s'épanouit au début $\mathrm{du} \mathrm{xIX}^{\mathrm{e}}$ siècle dans l'esthétique néoclassique, expliquant pour partie la présence massive de moulages d'œuvres antiques dans les écoles d'art d'Europe.

3 Parallèlement à cette internationalisation des références plastiques dans la seconde moitié $\mathrm{du} \mathrm{XVIII}^{\mathrm{e}}$ siècle se développent les premiers débats autour d'une nationalisation des écoles de peinture, que les guerres napoléoniennes nourrissent. «Ainsi, les écoles anglaise et allemande de peinture s'inventent dans la confrontation et la polémique 
avec l'école française ${ }^{1}$.» Pour les académies artistiques de chaque pays européen, il ne s'agit pas d'imiter l'antique mais d'en livrer une interprétation plastique propre à définir un art national. Ainsi, autour de la norme plastique antique se joue l'identité esthétique de chaque pays.

\section{L'influence de Paris et Rome dans la constitution de collections de plâtres d'antiques dans les académies d'Europe}

4 Le fonctionnement des académies européennes est souvent inspiré du modèle académique parisien en ce qui concerne le système d'émulation, tandis que les bases "scientifiques" de l'enseignement reprennent celles des premières académies italiennes fondées à la fin du XVI ${ }^{e}$ siècle - enseignement de la perspective, de l'anatomie et du dessin d'après le modèle vivant. Les statuts de la « Real Academia de Bellas Artes de San Fernando » de Madrid ${ }^{2}$, fondée en 1752, ceux de l'Académie impériale des beauxarts de Saint-Pétersbourg en 1757 ou ceux de l'Académie royale des beaux-arts de Copenhague, instituée en mai $1754^{3}$, attestent cette influence majeure.

Lors de la fondation des Académies en Europe, l'ouverture aux artistes étrangers est jugée nécessaire au progrès des arts et les artistes italiens et français sont fréquemment sollicités. Le peintre Louis-Michel Van Loo, qui travaille en France et en Espagne, est un membre fondateur de la Real Academia de Bellas Artes, créée en 1752. À Londres, la Royal Academy fondée en 1768 accueille des résidents étrangers comme le peintre Benjamin West, Giovanni Battista Cipriani ou Francesco Zuccarelli. Les professeurs circulent également dans toute l'Europe : à Copenhague, le directeur est le sculpteur français Jacques-François Saly, également membre de l'Académie de Florence et de Bologne, le professeur de sculpture Louis-Augustin Le Clerc, le professeur d'architecture Nicolas-Henri Jardin et son frère Louis-Henri, professeur de perspective ${ }^{4}$; à Saint-Pétersbourg, les peintres Louis-Joseph Le Lorrain et Louis-JeanFrançois Lagrenée dirigent un temps l'Académie, tandis que le sculpteur NicolasFrançois Gillet forme deux générations de sculpteurs en Russie. Enfin, à l'Académie de Vienne, dont la fondation remonte à 1692, le peintre autrichien Jacques Van Schuppen, neveu de Nicolas de Largillière, devient directeur de l'institution en 1725. Membre de l'Académie de Paris, il fait réformer l'institution à partir de 1735, au point d'en faire l'académie d'art la plus prisée de la sphère germanique au XVIII ${ }^{e}$ siècle. En territoire germanophone, le premier directeur de l'Académie de Berlin, le peintre Joseph Werner, était à Paris lors de la création de l'Académie royale et en reprend la structure administrative ${ }^{5}$. Le premier directeur de l'Académie de Dresde, fondée en 1764, est un peintre français, Charles-François Hutin. L'Académie recrute au XVIII ${ }^{e}$ siècle un corps enseignant international avec les Italiens Bernardo Bellotto, Giuseppe Camerata, Giovanni Battista Casanova et Lorenzo Zucchi ainsi que les Suisses Anton Graff et Adrian Zingg 6 . 


\section{La collection de moulages antiques à des fins pédagogiques : un geste inaugural}

6 Les collections de plâtres antiques forment souvent le fonds pédagogique initial des grandes académies européennes. Ces collections permettent à la fois d'incarner la culture humaniste élaborée par l'élite européenne depuis le $\mathrm{xvI}^{\mathrm{e}}$ siècle et de diffuser des références plastiques classiques communes, un langage commun des formes. "L'initiative de Louis XIV, qui avait commandé des copies en marbre de toutes les plus belles statues antiques d'Italie et qui s'était procuré une série de moulages en plâtre pour l'Académie de France à Rome, ne put être égalée par aucune des souverains suivants ${ }^{7}$. » Le prestige de la collection réunie à Rome est international : deux grandes collections de plâtres antiques constituées à la fin du XvIII ${ }^{e}$ siècle s'en inspirent, celle de l'Académie de Mannheim et celle, privée, du palais Farsetti à Venise.

7 En Espagne, dès 1650, le roi Philippe IV d'Espagne demande au peintre Diego Velazquez de faire des moulages de statues antiques à Rome, afin que ceux-ci servent de modèles pour des copies en bronze devant orner le Palais royal ${ }^{8}$. La dynastie des Bourbons montée sur le trône après la guerre de Succession d'Espagne, en quête de nouveaux modèles, se tourne vers Rome et la France afin de développer une nouvelle esthétique. Il s'agit de fonder le goût pour le " grand genre », volonté qui préside à la formation des collections de la Real Academia de Bellas Artes de San Fernando à Madrid. Les académies européennes emboîtent rapidement le pas. Dès 1682, l'Académie de La Haye se dote d'une série de plâtres d'antiques', tout comme l'Académie de Vienne (1692) qui reçoit en 1704 une collection de plâtres ${ }^{10}$ de Léopold $\mathrm{I}^{\mathrm{er}}$. Le prestige de la jeune Académie des beaux-arts de Saint-Pétersbourg repose sur sa collection de plâtres d'antiques envoyés de Rome dès 1758 , offerts par le comte Shuvalov ${ }^{11}$. Afin de fonder une esthétique nationale et moderne, il faut abandonner la tradition intellectuelle et artistique gréco-byzantine et se tourner vers " la grande manière ", à l'image de ce que l'Académie de Paris a réalisé. À ses débuts, l'Académie de Londres ne possède que quelques moulages d'antiques, et cherche activement de nouvelles sources pour en acquérir de nouveaux, notamment par le don de collectionneurs. Lorsqu'elle emménage en 1780 à New Somerset House, elle dispose de salles spécialement conçues pour le dessin d'après l'antique [fig. 1], à côté du splendide carton de la Sainte Anne de Léonard de Vinci ${ }^{12}$, seul dessin de maître de l'institution - la méthode pédagogique de son président, Joshua Reynolds, étant résolument opposée à la copie des dessins de maitres. 
Figure 1

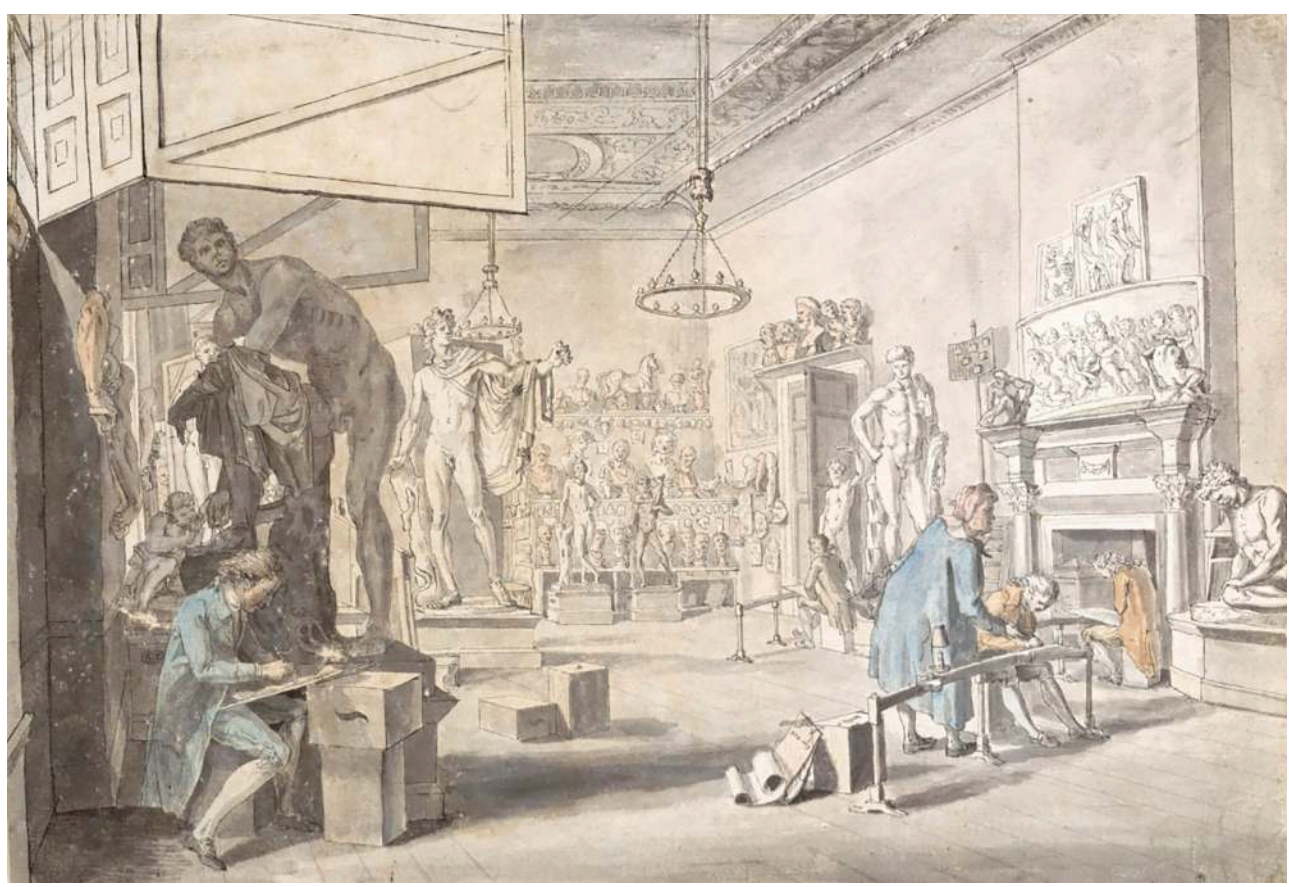

The Antique School at New Somerset House, Edward Francis Burney (1760-1848), vers 1780, dessin, plume et encre avec aquarelle sur papier vergé $(33,5$ × 48,5 cm) conservée à la Royal Academy of Arts (inv. 03/7484) à Londres.

Reproduction Royal Academy of Arts, Londres.

8 L'Académie royale des arts de Berlin, fondée en 1696, possède elle aussi de nombreux plâtres d'antiques ${ }^{13}$ [fig. 2], mais c'est surtout l'Antiksaal de l'Académie de Mannheim, ouverte de 1758 à 1804, qui devient célèbre grâce aux visites des personnalités qui fondent le romantisme allemand, au premier rang desquelles se trouve Johann Wolfgang Goethe. L'Académie de Copenhague possède dès son inauguration en 1754 une petite collection de plâtres, d'abord installée au château royal de Charlottenborg puis transférée à l'Académie ${ }^{14}$ [fig. 3]. Ce collectionnisme s'intensifie tout au long du $\mathrm{XVIII}^{\mathrm{e}}$ siècle à la faveur de la réflexion internationale sur les œuvres antiques, qui contribue à diffuser un ensemble de valeurs esthétiques, dites néoclassiques, en réalité très variées dans leurs conceptions et dans leurs expressions. 
Figure 2

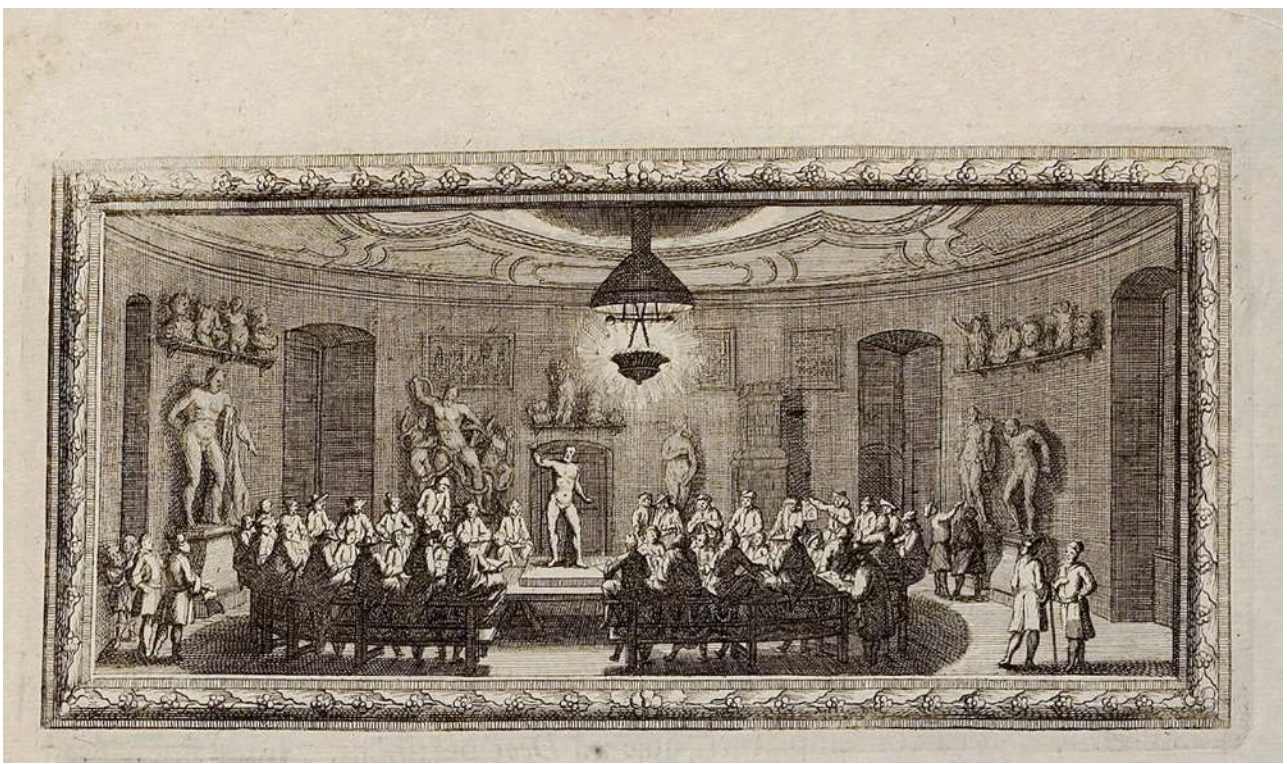

9 Aktsaal der Kunst-Academie zu Berlin, Christoph Weigel (1654-1725), d'après Erwesten (1649-1711), 1697. Gravure extraite de Lorenz Beger, Thesaurus brandenburgicus selectus, sive gemmarum et numismatum graecorum in cimeliarchio electorali Brandenburgico, elegantiorum series, commentaris illustratae a L.Begero, III, Coloniae Marchicae, Berlin, Stiftung Preußische Schlösser und Gärten Berlin-Brandenburg, 1701, p. 217.

Reproduction Bibliothèque universitaire de Heidelberg. 


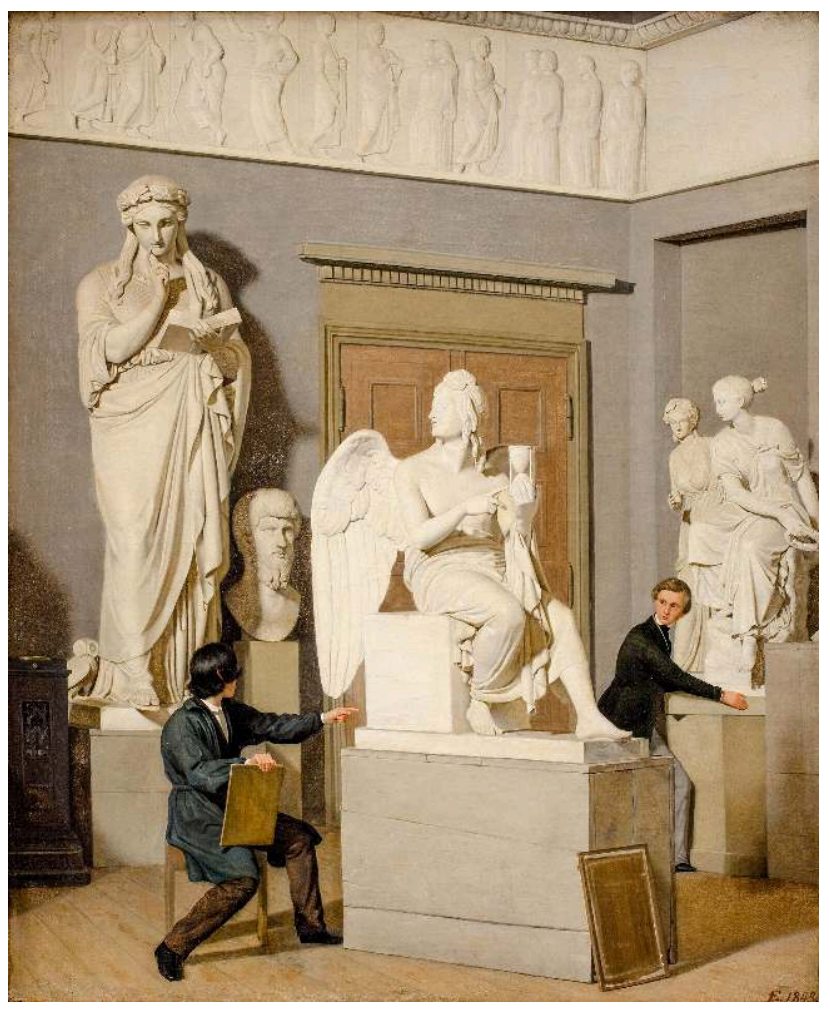

Fra Kunstakademiets figursal (De l'Académie des Beaux-Arts), Julius Exner (1825-1910), 1843, huile sur toile $(60 \times 49.5 \mathrm{~cm})$ conservée à la Statens Museum for Kunst (inv. KMS3110) à Copenhague, Danemark.

Reproduction Statens Museum for Kunst.

\section{Les séjours à Rome et à Paris}

Un élément important du système d'émulation de l'académie parisienne est repris dans de nombreuses académies européennes : le séjour à Rome financé pour les lauréats du "Prix de Rome ", artistes sur lesquels la nation fonde de grands espoirs. Rome n'est pas la seule destination jugée essentielle en Europe : Paris est aussi un lieu de destination privilégié par les académies européennes. L'Académie de Madrid finance de manière irrégulière des séjours à l'étranger depuis 1758, notamment à Paris et à Rome. À SaintPétersbourg, on considère que les séjours peuvent se faire aussi bien à Rome qu'à Paris ${ }^{15}$, l'ascendant de l'art français sur les sculpteurs russes étant depuis longtemps très fort, que ce soit par l'enseignement du sculpteur Gillet à l'Académie ou leur perfectionnement dans les ateliers de Lemoyne, Pigalle d'Allegrain, Pajou, Houdon. Le sculpteur Féeodosevich Chtechéedrine conseille "d'envoyer tous les jeunes élèves à Paris, parce qu'ils y trouvent une grande émulation [...] et de finir par Rome parce qu'on y trouve [...] les grands modèles éternels qui inspirent un autre genre d'émulation quand on a commencé à se sentir et à pénétrer tous les secrets de l'art ${ }^{16}$ ». Rome et Paris offrent un environnement favorable aux capacités artistiques selon Denis Diderot : «Croyez-vous que les beaux-arts puissent avoir aujourd'hui à Neuchâtel ou à Berne le caractère qu'ils ont eu autrefois dans Athènes ou dans Rome ou même celui qu'ils ont sous nos yeux à Paris ${ }^{17}$ ? » Si Raphaël Mengs «fait des prodiges », c'est bien 
parce qu'il est à Rome : « Séparez-le des grands modèles, enfermez-le à Breslaw, et nous verrons ce qu'il deviendra.»

11 S'il faut se garder de surévaluer l'attractivité de Rome et de Paris dans la circulation des acteurs artistiques européens ${ }^{18}$, la valeur symbolique du voyage à Rome est immense. Expérience esthétique indispensable, le «Grand Tour » et la copie de chefs-d'œuvre demeurent l'activité essentielle de l'Académie de France à Rome. Cette symbolique culmine avec le propos attribué au peintre Jacques-Louis David, à qui le séjour à Rome a " ouvert les yeux ». Et ce n'est pas seulement l'antique que désigne le peintre par cette référence, mais aussi la peinture de Raphaël : «Le goût, les habitudes, l'intelligence même, avaient chez moi quelque chose de gaulois, de barbare, dont il fallait qu'elle se dépouillât, pour arriver à l'état d'érudition et de pureté dans lequel on admire les stanze de Raphä̈l ${ }^{19}$.» Toute l'avant-garde artistique séjourne à Rome: les artistes y demeurent continûment présents du milieu du XvIII ${ }^{\mathrm{e}}$ siècle au début du XIX ${ }^{\mathrm{e}}$ siècle, en dépit de l'instabilité politique de l'Europe, des débuts de la Révolution aux guerres napoléoniennes.

12 L'historien de l'art et archéologue Johann Joachim Winckelmann fait de Rome le centre stratégique de son combat pour l'idéal de la Grèce antique, secondé par le peintre et écrivain d'art allemand Anton Raphaël Mengs qui découvre que quantité de statues des collections pontificales ne sont pas des originaux grecs mais des copies romaines réalisées en marbre italien. Cette controverse passionne l'Europe entière, et particulièrement l'élite des artistes européens séjournant à Rome. La renommée de Winckelmann et de Mengs attire un nombre croissant d'artistes et écrivains allemands comme Goethe, qui vient visiter en 1786 la galerie des plâtres d'antiques de l'Académie de France à Rome ${ }^{20}$. Pendant la Révolution, la Ville éternelle devient terre d'asile pour « une quantité prodigieuse d'artistes ${ }^{21}$ » avant 1793 , puis une plaque tournante de toutes les migrations européennes.

D'importantes personnalités artistiques liées à la sensibilité néoclassique, "fruit d'une réflexion savante et archéologique sur les origines de l'européanité22 », développent une carrière dans plusieurs pays européens et contribuent à uniformiser le culte de l'antique. Mengs, né en Bohême, séjourne alternativement à Dresde, Rome et Madrid. Son influence dans le milieu académique européen est considérable sur le plan des collections, et ses écrits sont indifféremment rédigés en espagnol, en italien ou en allemand. Il lègue à l'Académie de Madrid les nombreux plâtres antiques qu'il possédait en 1778, tandis que Dresde fait l'acquisition de l'autre partie de sa collection en $1783^{23}$ lors de sa succession ${ }^{24}$. Un autre exemple significatif est celui d'Angelica Kauffmann, peintre autrichienne née en Suisse, qui séjourne plusieurs fois à Rome, où elle rencontre Benjamin West, Jacques-Louis David, Johann Winckelmann et Anton Raphaël Mengs. Elle part ensuite à Londres, où elle rencontre Joshua Reynolds et son futur mari, le peintre vénitien Antonio Zucchi, avant de revenir à Rome dans les années 1780. Elle installe son atelier dans l'ancienne maison de Mengs, qui devient le lieu de rencontre des artistes ainsi que de leurs commanditaires aristocrates. Tout au long de sa carrière, Angelica Kauffmann s'investit dans plusieurs académies européennes: d'abord en Italie, à l'Académie de Bologne et à l'Accademia di San Luca de Rome, puis devient un des membres fondateurs de la Royal Academy en 1768 [fig. 4]. Ces parcours, auxquels il serait possible d'en ajouter beaucoup d'autres, montrent à quel point les artistes et les modèles circulent dans l'Europe des Académies à la fin du XVIII e siècle, à partir de Rome et de Paris. 


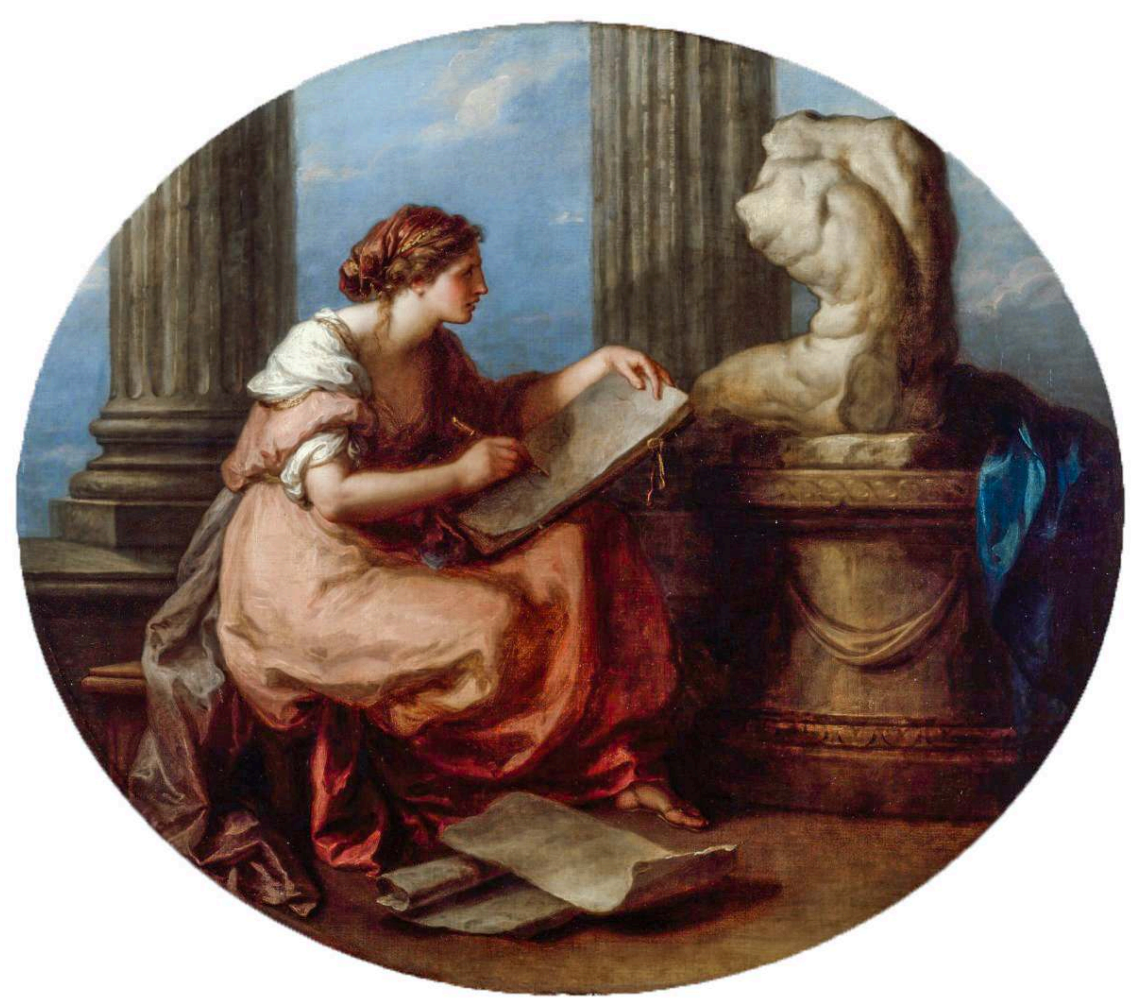

Le Dessin, Angelica Kauffmann, 1778-80, huile sur toile $(126$ × 148 cm) conservée à la Royal Academy of Arts (inv. 03/1129), Londres.

Reproduction John Hammond (Royal Academy of Arts).

\section{De nouveaux modèles pédagogiques : des collections de plâtres diversifiées, des formes nationales}

Depuis la fin du $\mathrm{XVIII}^{\mathrm{e}}$ siècle, et parallèlement à la diffusion européenne des idées néoclassiques, le besoin d'une définition de l'art selon des spécificités nationales se fait jour un peu partout en Europe, avant même les bouleversements politiques et territoriaux créés par la Révolution et les guerres napoléoniennes. La science antiquaire alimente désormais les tensions politiques, avec un musée Napoléon rassemblant les plus célèbres antiques, indéniable réussite muséographique à la fois admirée et honnie. Dans les collections académiques, il est temps de proposer des modèles nationaux propres à affirmer le tempérament de chaque nation.

\section{L'influence du musée Napoléon sur le patrimoine pédagogique des écoles d'art au XIX ${ }^{\mathrm{e}}$ siècle : enrichissement des collections de plâtres d'antiques}

La "fête de la Liberté et des Arts» de 1798, qui met en scène l'arrivée du buste d'Homère, de l'Apollon du Belvédère, du Laocoon et de la Vénus Médicis, fait de Paris le nouveau centre artistique de l'Europe [fig. 5]. L'arrivée de la Vénus Médicis en 1803, puis des antiques de la collection Borghèse en 1808, complète la collection rassemblée au 
musée Napoléon, qui marque toute une génération d'artistes jusqu'en 1815. Elle suffit à légitimer le séjour parisien des artistes de toute l'Europe au moment où l'Antiquité est le modèle absolu. Le Musée produit et vend des moulages en plâtre de ses trésors antiques non seulement sur son propre territoire, mais dans l'Europe entière ${ }^{25}$, devenant un précurseur des sociétés commerciales spécialisées dans ce type de production qui connaîtront un grand succès tout au long du XIX ${ }^{e}$ siècle.

Figure 5

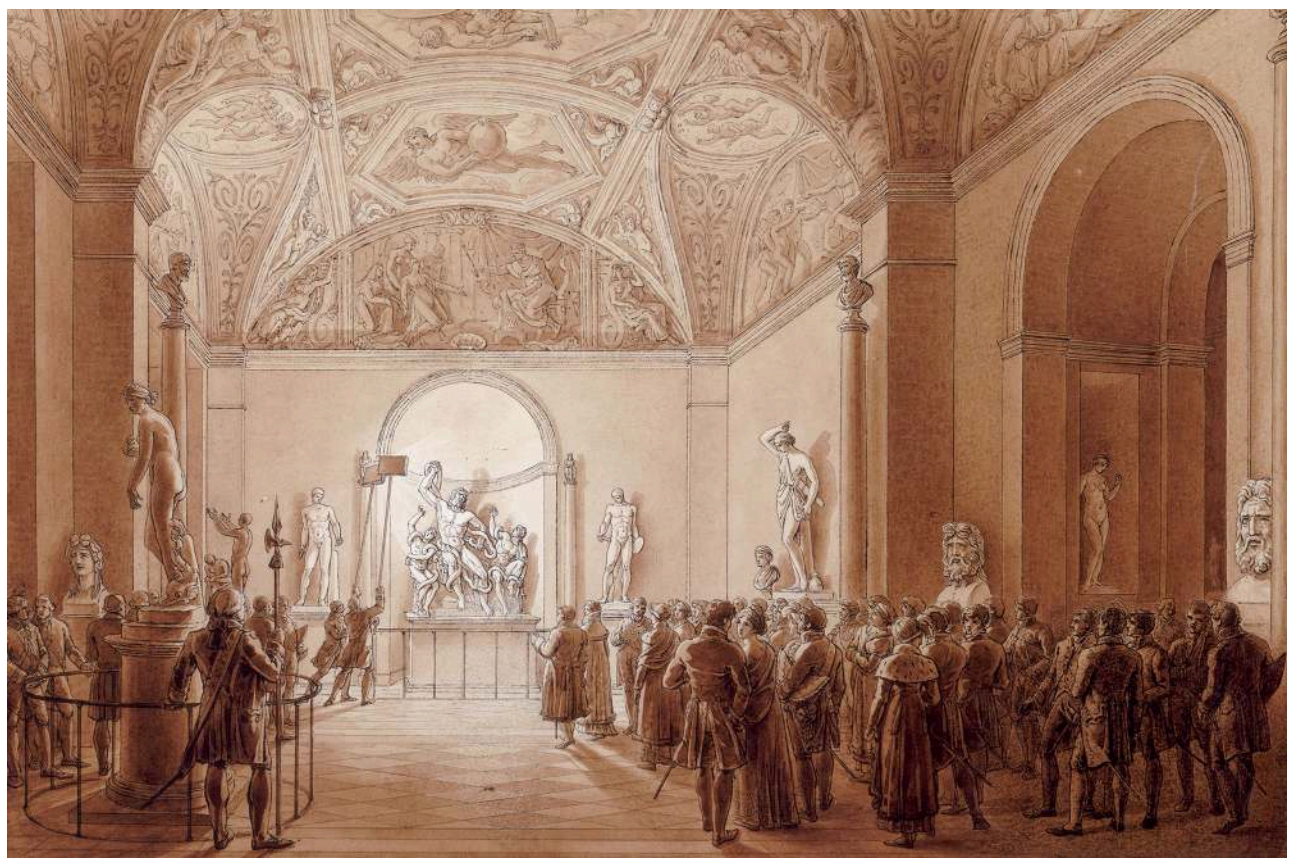

Visite aux flambeaux faite par l'Empereur et l'Impératrice. Salle du Laocoon du Louvre, Benjamin Zix (1772-1811), vers 1810, encres brune et grise, lavis brun et dessin à la plume $(26 \times 29 \mathrm{~cm})$. Dessin conservé au musée du Louvre (inv. 33406-recto).

Reproduction Gérard Blot (RMN-Grand Palais).

Une série entière de trente-six caisses est ainsi envoyée à l'Académie royale des arts de La Haye ${ }^{26}$. À Saint-Pétersbourg, l'empereur Paul I ${ }^{\text {er }}$ donne à l'Académie une «riche collection de beaux moules des plus fameux chefs-d'œuvre de l'Antiquité, autrefois appartenant à la maison Farsetti à Venise et transportée de là en 1800 à SaintPétersbourg; la plus grande et meilleure partie de cette collection fut placée à la classe afin d'avoir toujours des beaux modèles sous les yeux ${ }^{27} »$. En Espagne, le prestige des plâtres d'antiques de la collection Mengs est immense et les artistes espagnols séjournant à Rome continuent d'envoyer des plâtres d'antiques à Madrid afin d'enrichir les collections de l'Académie. L'inventaire de 1804 en témoigne: les plus célèbres modèles antiques sont non seulement présents, de même que des copies d'œuvres d'art antique des fouilles d'Herculanum ${ }^{28}$. En raison de la fusion des deux académies, la collection de l'Antikensaal de Mannheim est transférée à l'Académie de Munich en 1807, et continue de fasciner les jeunes artistes. L'enseignement à l'Académie de Berlin se réforme sous la direction du peintre et sculpteur Johann Gottfried Schadow à partir de 1816. Il y constitue une collection élargie de plâtres antiques ${ }^{29}$, car avec l'arrivée des marbres de l'Acropole en 1816 à Londres, toutes les académies cherchent à acquérir des moulages de ces frises ${ }^{30}$. En France, la collection pédagogique de plâtres d'antiques s'enrichit encore avec plusieurs dons relatifs à l'ornement effectués en 1798 puis, au 
début du XIX ${ }^{e}$ siècle, avec la collection du comte de Choiseul-Gouffier qui rejoint en partie le fonds de l'école ${ }^{31}$.

\section{La reprise en main nationale de l'enseignement des écoles d'art et la mise en avant de nouveaux modèles}

17 À partir de 1820, le voyage en Italie décline au profit des expéditions menées dans le nord de l'Europe, notamment en Écosse et en Angleterre, ainsi que de celles que les Français ou les Allemands effectuent dans leur propre pays à des fins patriotiques. Si la curiosité antiquaire demeure vivace en Europe, elle se déploie sur les "antiquités nationales », prenant en compte des antiquités du Moyen Âge ou celles d'un passé plus lointain mais local.

Dans des circonstances politiques agitées après l'invasion des troupes napoléoniennes, l'Académie de Madrid freine à plusieurs reprises l'envoi d'artistes à l'étranger au début $\mathrm{du} \mathrm{XIX}^{\mathrm{e}}$ siècle. Les théories sur l'enseignement de Mengs sont critiquées dès 1803, mettant en cause, l'enseignement exclusif d'après les sculptures antiques ${ }^{32}$. L'étude assidue d'après le modèle vivant, comme à l'époque de Goya ${ }^{33}$ est préconisée, et dessins ou académies peintes deviennent de plus en plus naturalistes. La même année, le roi Charles IV confie à la Real Academia de la Historia le soin d'inventorier et de protéger les monuments hispaniques ${ }^{34}$. Le choix des sujets de concours tend à valoriser l'histoire de l'Espagne. Les académiciens estiment que les artistes peuvent se former à Madrid, et que les œuvres conservées dans les collections madrilènes suffisent à leur formation ${ }^{35}$. La collection de plâtres s'est diversifiée avec les derniers envois des pensionnaires à Rome : bas-reliefs de sculpteurs de la Renaissance et de la période baroque, comme Michel-Ange, Le Bernin ou Alessandro Algardi, mais aussi des moulages des reliefs des portes du baptistère de Florence ${ }^{36}$. Dans les années 1836, avec l'arrivée de la collection Valparaíso à l'Académie, albums d'estampes et de dessins des principales écoles de gravure européennes des $\mathrm{XVI}^{\mathrm{e}}, \mathrm{XVII}^{\mathrm{e}}$ et $\mathrm{XVIII}^{\mathrm{e}}$ siècles viennent enrichir les références plastiques $^{37}$. 


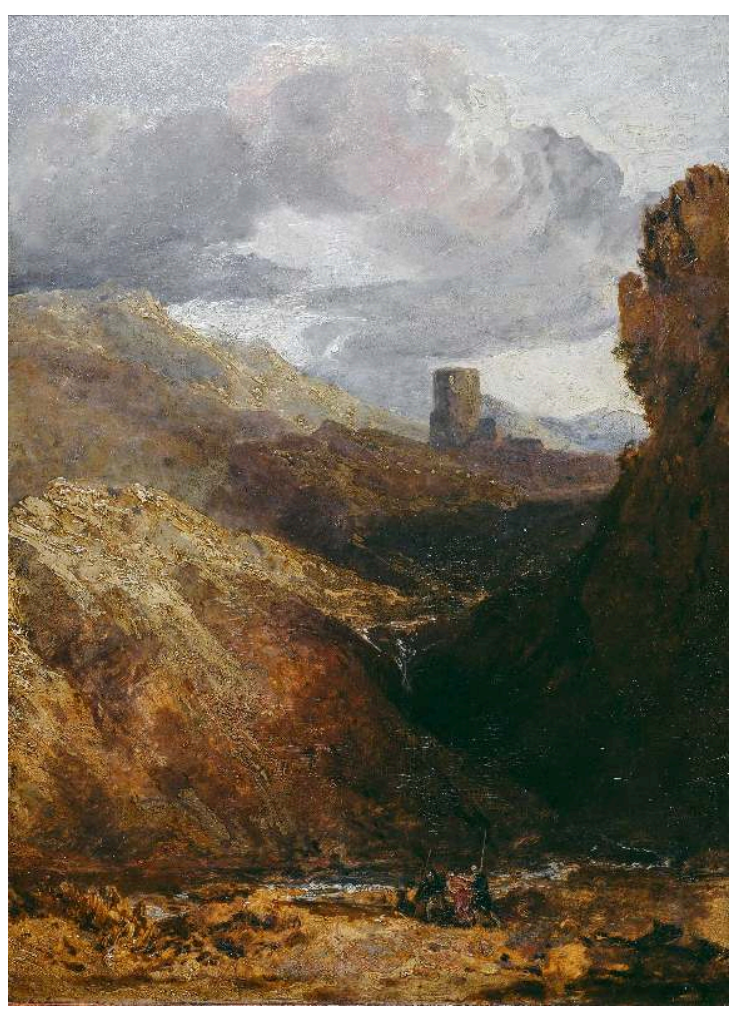

Dolbadarn Castle, North Wales, Joseph Mallord William Turner, 1800, huile sur toile $(119,4 \times 90,2 \mathrm{~cm})$ conservée à la Royal Academy of Arts (inv. 03/1383) à Londres.

Reproduction Prudence Cuming Associates Limited (Royal Academy of arts).

En Angleterre, un discours britannique sur l'art s'est élaboré tout au long du XVIII ${ }^{\mathrm{e}}$ siècle, du comte de Shaftesbury, théoricien du beau, à Reynolds, et trouve son expression artistique dans le succès international des œuvres de Joshua Reynolds et de Thomas Gainsborough puis de John Constable et de William Turner [fig. 6]. La Royal Academy, qui avait été fondée en 1768 de manière à favoriser la rencontre entre le goût du public et celui d'un art national correspondant aux critères académiques, parvient à ses fins au début du xix ${ }^{e}$ siècle, avec la reconnaissance européenne d'une école spécifiquement anglaise ${ }^{38}$. Certes, William Blake ou George Romney se tiennent éloignés de l'institution, mais en laissant les artistes libres dans leurs interprétations plastiques de la culture empiriste du pays, prônant la découverte et l'observation attentive des phénomènes de la nature, y compris la nature humaine ${ }^{39}$, l'art anglais s'est construit une identité, notamment à travers le portrait et la peinture de paysage, mais aussi à travers la veine fantastique. Le rapport à l'œuvre d'art devient direct et émotionnel, ne nécessitant que peu de bagage culturel préalable, en ce sens précurseur de la modernité. Dans les collections de la Royal Academy, ce fait se traduit par la présence de dessins d'après Michel-Ange, artiste régulièrement mis à l'écart à l'Académie de Paris, mais aussi par la conception de modèles anatomiques nationaux, qui matérialisent le retour à l'école de la nature et au primat de l'observation. Le plus spectaculaire est celui réalisé en 1801 par le sculpteur néoclassique Thomas Banks [fig. 7], un écorché crucifié, dans le but de trancher un débat artistique au sujet des incorrections anatomiques des représentations traditionnelles. L'observation de la nature prime, comme elle a toujours primé chez les Anglais, ce qui n'empêche en rien la 
Royal Academy de continuer à acquérir des moulages d'antiques, notamment auprès de la firme londonienne D. Brucciani \& Co à partir de 1837. Les quelques dessins d'après les plâtres d'antiques conservés montrent qu'ils devaient être accompagnés d'un schéma anatomique. Le vrai sésame se trouve en bas du dessin jugé "for admission for the life », c'est-à-dire " admis dans la classe du modèle vivant », plébiscitée par les étudiants ${ }^{40}$ au grand regret du peintre américain Benjamin West lors de son directorat.

Figure 7

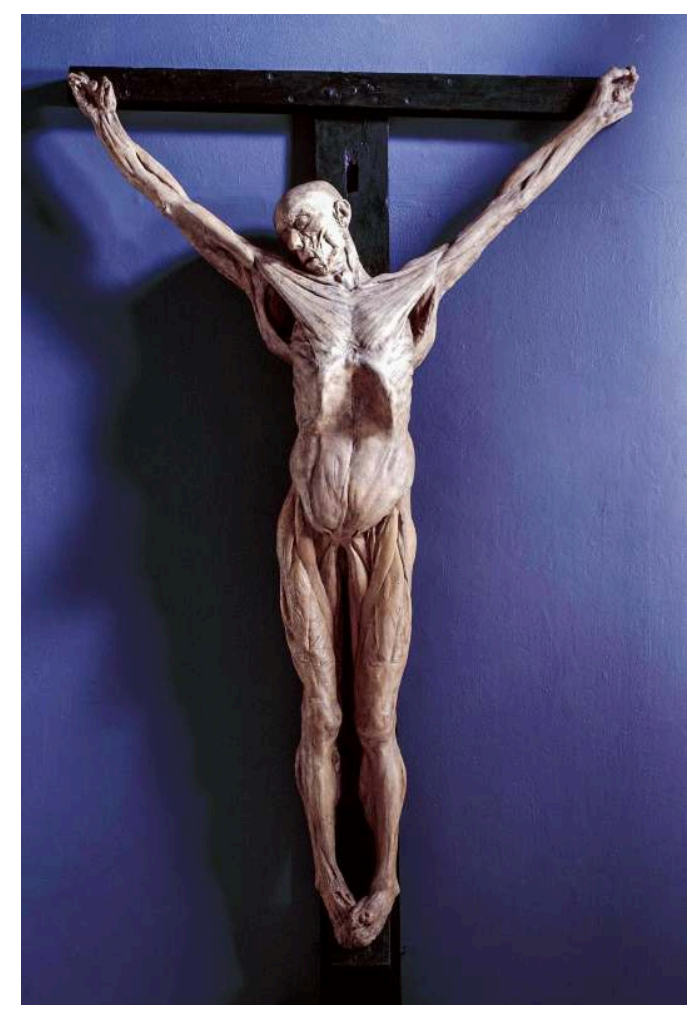

Anatomical Crucifixion, Thomas Banks (1735-1805), 1801, moulage en plâtre sur cadavre (231,5 x 141 cm) conservé à la Royal Academy of Arts (inv. 03/1438), Londres.

(c) Photographe inconnu (Royal Academy of Arts).

À Saint-Pétersbourg, les séjours de six années à Paris et Rome, en passant par Berlin, Dresde et Munich, continuent d'être financés, dans l'objectif d'assimiler toutes les formes de l'art occidental et antique, mais le but est bien de concevoir une esthétique nationale. Le premier artiste russe à être internationalement reconnu, le peintre Karl Brioullov, triomphe enfin à Rome et Paris avec Le Dernier Jour de Pompéi (SaintPétersbourg, Musée russe), qui remporte une médaille au Salon parisien de 1834 [fig. 8]. Une salle séparée lui est dédiée à l'Académie pour que le public puisse admirer le tableau ${ }^{41}$. L'école russe est née aux yeux des Européens. Le conseil des professeurs de 1836 n'a plus qu'à insister pour choisir davantage de thèmes nationaux comme sujet de fin d'études ${ }^{42}$. Si les plâtres antiques sont toujours utilisés pour la formation, les élèves redécouvrent les peintures de la donation Shuvalov, avec ses riches exemples flamands ou hollandais, fins observateurs de la nature, et si opposés au "grand goût " que défendait Denis Diderot lors de son séjour à l'Académie de Saint-Pétersbourg ${ }^{43}$. 
Figure 8

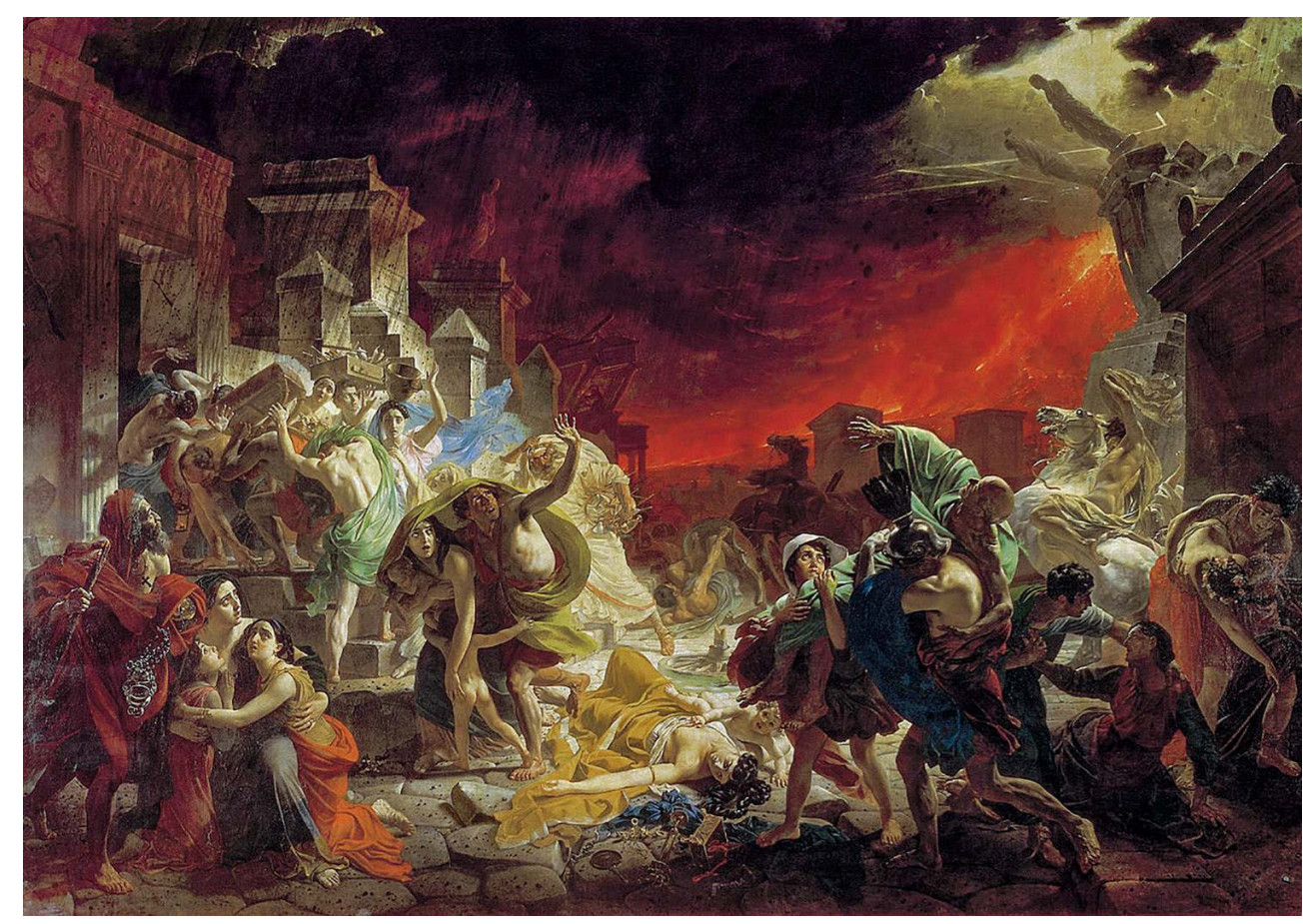

Le Dernier Jour de Pompéi, Karl Briullov, 1830-1833. Ancienne collection de l'Académie, déposé au Musée Russe de Saint Pétersbourg.

Reproduction Musée Russe de Saint Pétersbourg.

21 À Paris, les collections prennent elles aussi une couleur nationale. Une ordonnance royale de 1816 affecte l'ancien musée des Monuments français d'Alexandre Lenoir à l'École royale et spéciale des beaux-arts, qui se trouve ainsi dotée de modèles ornementaux nationaux d'une grande richesse. La place du modèle antique demeure très importante, mais aboutit parfois à des effets paradoxaux comme Jean-Galbert Salvage, avec sa tête d'Apollon en définitive défigurée [fig. 9]. Au milieu du XIX siècle, le modèle antique se voit de plus en plus contesté par les artistes modernes pourtant issus de l'école [fig. 10]. Le peintre Eugène Delacroix écrit en 1853 : « Ils croient qu'ils ont fait le beau quand ils ont répété à satiété les nez droits, les draperies en tuyaux de l'antique [...]. L'imagination des hommes [...] sera-t-elle enchaînée à l'admiration unique de ces types? Ils sont sublimes d'ailleurs; ce que je réprouve, c'est l'imitation unique ${ }^{44}$.» Delacroix ne voyage pas en Italie, ni en Grèce. Il accompagne une mission diplomatique en Algérie et choisit en 1839 de visiter les Flandres pour admirer les peintures de Rubens. 


\section{Figure 9}

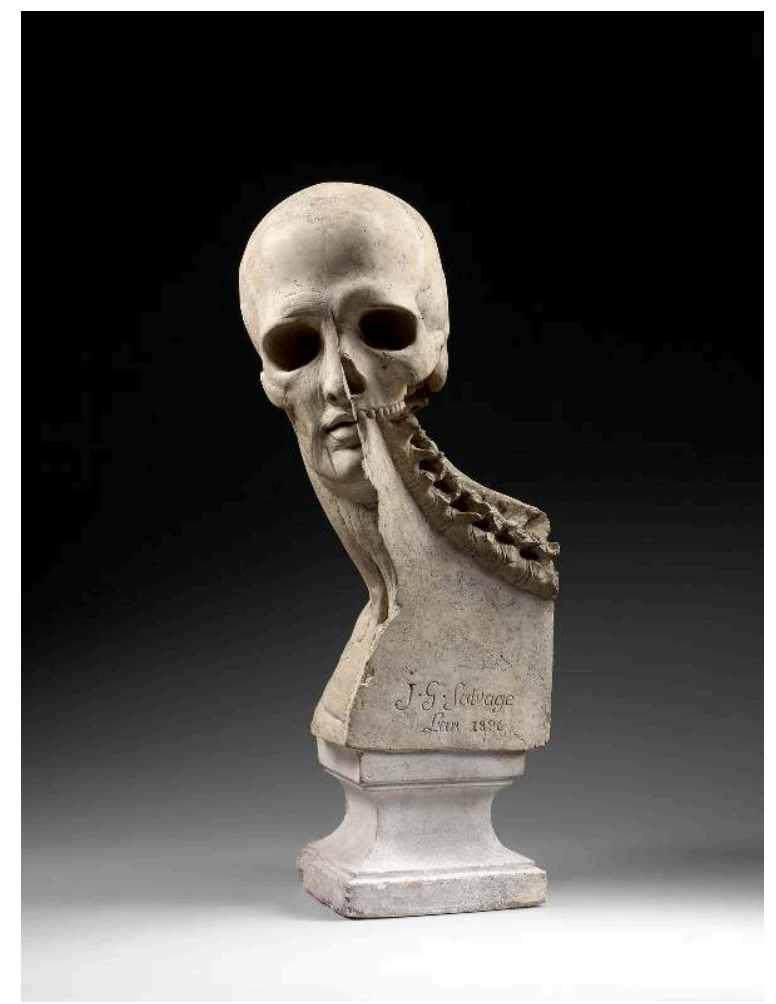

Apollon du Belvédère, Jean-Galbert Salvage (1772-1813), demi-écorché de la tête et du cou, moulage en plâtre $(67 \times 34 \mathrm{~cm})$ conservé à l'École nationale supérieure des beaux-arts (inv. MU11923), Paris.

(c) Photographe inconnu (RMN-Grand Palais). 


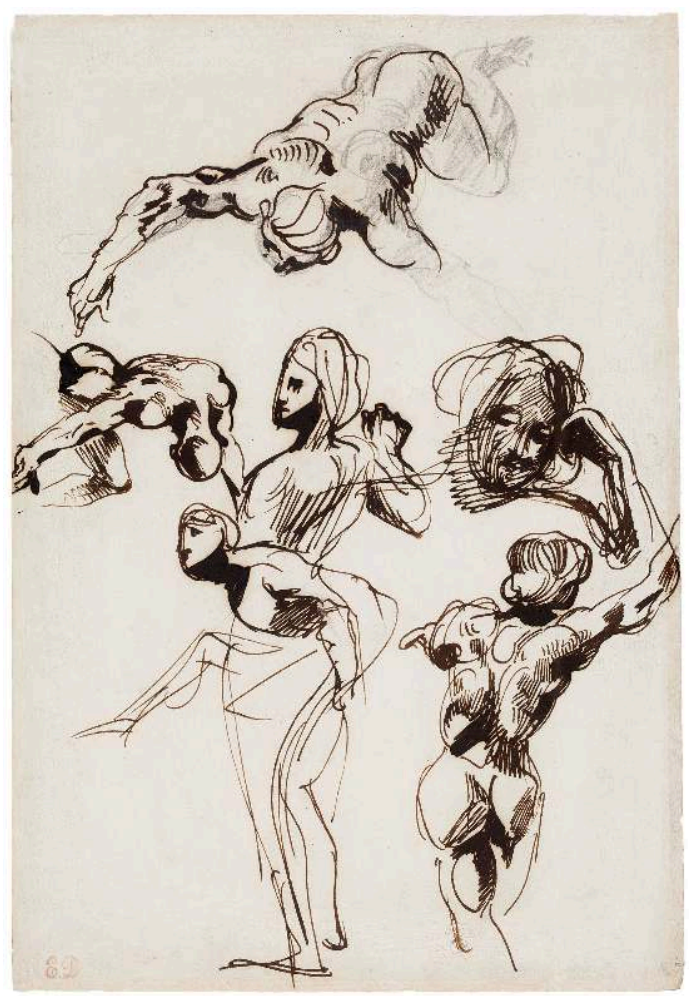

Feuille d'étude, Eugène Delacroix (1798-1863), dessin à la plume $(28,5$ x 19,5cm) conservé à l'École nationale supérieure des beaux-arts (inv. EBA10701), Paris.

Reproduction RMN-Grand Palais.

L'affirmation d'une école allemande des arts ne se fait pas dans un premier temps contre le modèle antique, mais en accord avec une certaine vision de l'art antique, sensible et créative, loin du style français de l'école de Jacques-Louis David, qualifié généralement de style maniéré et théâtral. Très vite cependant, l'affirmation d'un art national passe par le choix de nouveaux modèles. Le rayonnement européen des Nazaréens, artistes allemands installés à Rome, en est la première manifestation ${ }^{45}$. Ces artistes, en rupture avec l'enseignement rigide dispensé à l'Académie de Vienne à partir de l'antique, veulent retrouver le purisme italien, une unité morale et spirituelle totale, en s'inspirant d'Albrecht Dürer et de Raphaël. L'Académie de Berlin, qui leur consacre une exposition en 1818, conserve encore des œuvres de cette école. Certains artistes nazaréens vont alors être appelés à diriger des académies comme Peter von Cornelius à Munich ou Philipp Veit, nommé directeur de l'école de peinture et directeur des collections d'art à Francfort en 1830.

Le peintre aujourd'hui le plus connu de l'école allemande, Caspar David Friedrich, s'est formé à l'Académie royale de Copenhague, qui enseigne le dessin d'après le modèle antique. L'étudiant se montre très critique avec l'esthétique classique, qu'il théorise dans de rares textes : « Le peintre ne doit pas seulement peindre ce qu'il voit devant lui, mais ce qu'il voit en lui. S'il ne voit rien en lui, qu'il cesse aussi de peindre ce qu'il voit devant lui ${ }^{46}$.» Il devient membre de l'Académie de Berlin (1810) puis de Dresde (1816) mais n'en devient jamais professeur. Son influence tient davantage à la présence des œuvres dans les collections ouvertes au public, comme à Dresde, qui conserve une centaine d'œuvres du peintre. L'influence dans le milieu académique se fait sentir avec 
les peintres de l'école de Düsseldorf qui, dans les années 1830-1840, remettent en question la polarité entre réalisme et idéalisme, les références et les modèles de l'identité artistique nationale ${ }^{47}$. Si les plus complètes collections de plâtres d'antiques se trouvent en Allemagne, les œuvres des jeunes artistes sont manifestement davantage marquées par les sensibilités romantiques et la recherche de l'expression que par l'esthétique classique [fig. 11].

Figure 11

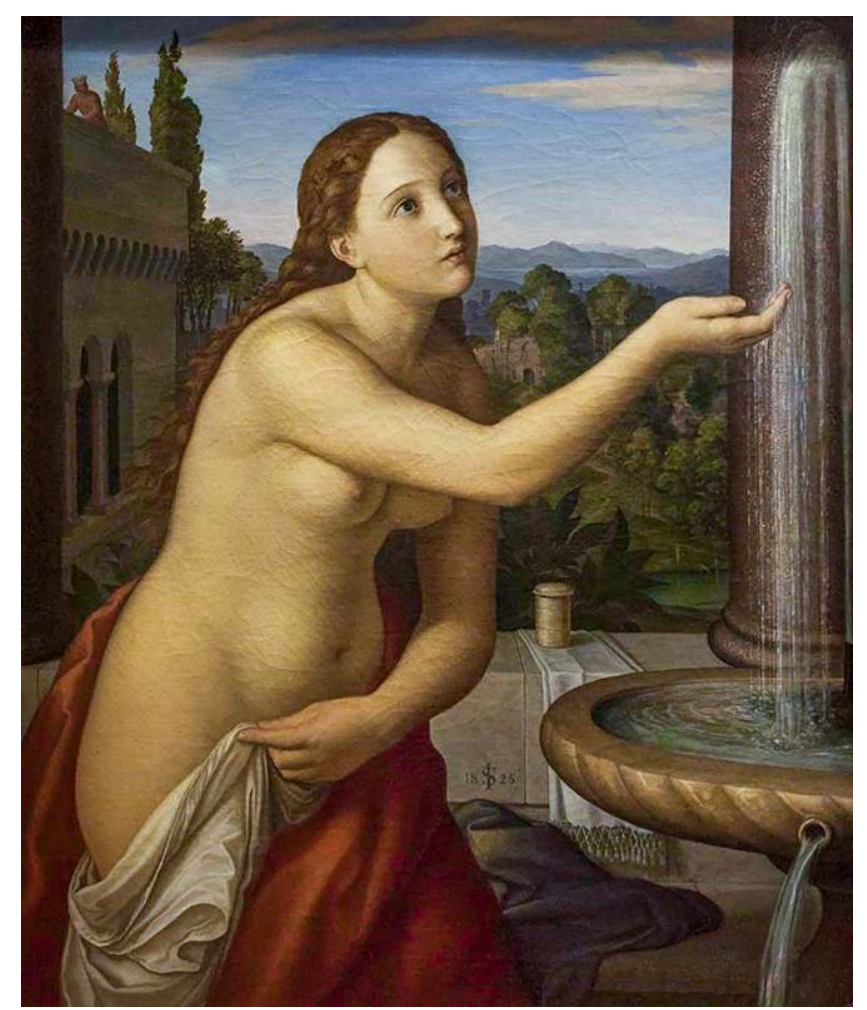

Béthsabée, Julius Schnorr von Carolsfeld,1820. Peinture conservée à l'Akademie der Künste, Berlin. Reproduction Akademie der Künste, Berlin.

\section{De l'effet inattendu des collections de plâtres dans les écoles d'art : regard plastique et revival}

\section{Discours historique et survivances pédagogiques : les raisons d'être des collections patrimoniales relatives à l'antique}

24 Au long du xix siècle, le plâtre antique se voit accaparé par le discours historique : nombre de collections de moulages d'antiques rejoignent alors des institutions muséales au fil du $\mathrm{XIX}^{\mathrm{e}}$ siècle car ils sont destinés à montrer l'histoire de la sculpture avec le moins de lacunes possible, comme c'est le cas de la collection de l'Académie de Copenhague, transférée en grande partie en 1893 au Musée national. Le chemin s'inverse parfois: la galerie des Plâtres au Louvre est supprimée en 1857 et ses moulages reviennent à l'École des beaux-arts de Paris.

La présentation chronologique s'impose progressivement. À Bonn, à l'époque de Goethe, le conservateur a disposé les plâtres en fonction de critères esthétiques, mais 
quarante années plus tard, son successeur impose une présentation chronologique. À Berlin, Carl Bötticher a suscité les protestations amères de ses collègues quand il a abandonné la chronologie, en 1868 , et a privilégié une présentation iconographique ${ }^{48}$. Si les collections deviennent de plus en plus complètes dans la seconde moitié du $\mathrm{XIX}{ }^{\mathrm{e}}$ siècle, et offrent la possibilité d'être exposées de manière plus cohérente, il est significatif de voir les artistes préférer l'ordonnancement des cabinets d'amateur à celui des archéologues, comme le montrent les vues imaginaires du British Museum par James Stephanoff [fig. 12].

Figure 12

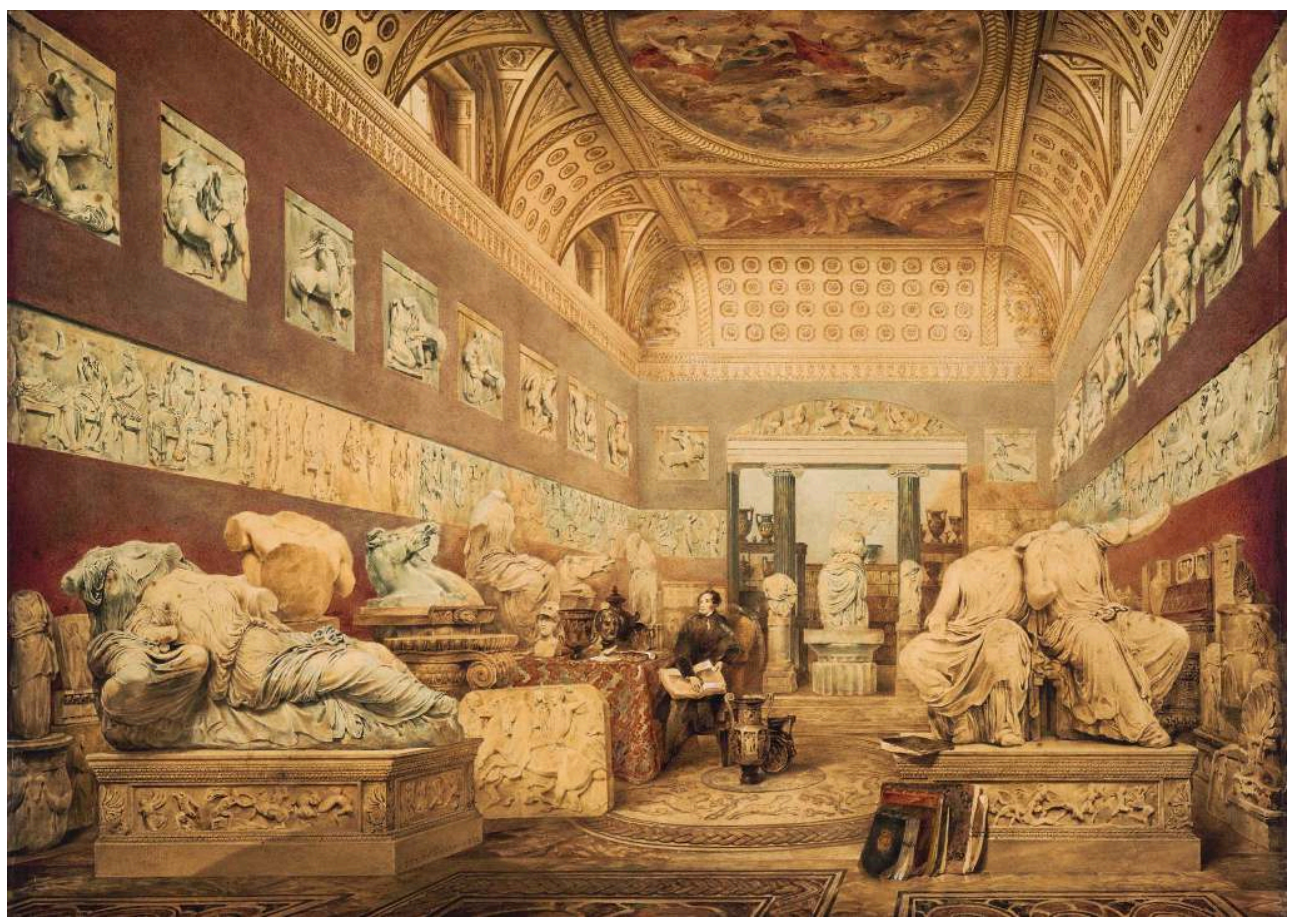

The Virtuoso, James Stephanoff, 1833, dessin à l'aquarelle $(51 \times 72,2 \mathrm{~cm})$ conserve au British Museum (inv. 1934,0113.1), Londres.

Reproduction Trustees of the British Museum.

L'intérêt demeure vif dans les écoles d'art en ce qui concerne les collections en plâtre, mais l'antique est loin d'en être le seul sujet. La rêverie plastique a supplanté la norme esthétique. Au milieu des forêts de plâtres, l'attrait devient celui d'un livre ouvert en grandeur naturelle, qui permet aussi de juxtaposer des œuvres d'art de différents pays et périodes. Du reste, la collection que Mengs avait constituée allait déjà dans ce sens. Ce qui a stimulé son travail théorique, c'est la variété des solutions plastiques adoptées par les artistes antiques. Au-delà des plus célèbres statues antiques, le nombre important de torses montre son intérêt pour les fragments non manipulés ou retravaillés, de même que le nombre de portraits anciens montre non la recherche de séries complètes mais celle de têtes expressives des deux sexes et de tous âges. Le mélange des époques est aussi frappant parmi les plâtres conservés à Dresde et à Madrid : nombre de plâtres de la Renaissance ou de la période baroque, qui ont tous en commun leur expressivité du relief ou de la figure ${ }^{49}$. Ce n'est pas la seule plastique antique qui est appréciée mais un ensemble de formes moulées, éclectique au fil des 
années, livré aux méditations de l'artiste en formation au gré de muséographies changeantes.

\section{Une Antiquité inattendue dans la deuxième moitié du XIX ${ }^{e}$ siècle}

Ce patrimoine pédagogique, commun à toute l'Europe, explique pour partie le singulier mouvement de représentations antiques modernisées qui saisit l'Europe dès le milieu du XIX ${ }^{e}$ siècle dans le domaine de la peinture. Les collections des académies en portent encore la trace, conservant nombre d'œuvres d'étudiants ou de professeurs, qui, entre 1847 et l'orée $\mathrm{du} \mathrm{xx}^{\mathrm{e}}$ siècle, se sont inventé une Antiquité désirable et vendeuse. Le public européen apprécie en effet ce genre pictural évocateur de l'histoire des mœurs gréco-romaines, qualifié aujourd'hui d'académique.

Figure 13

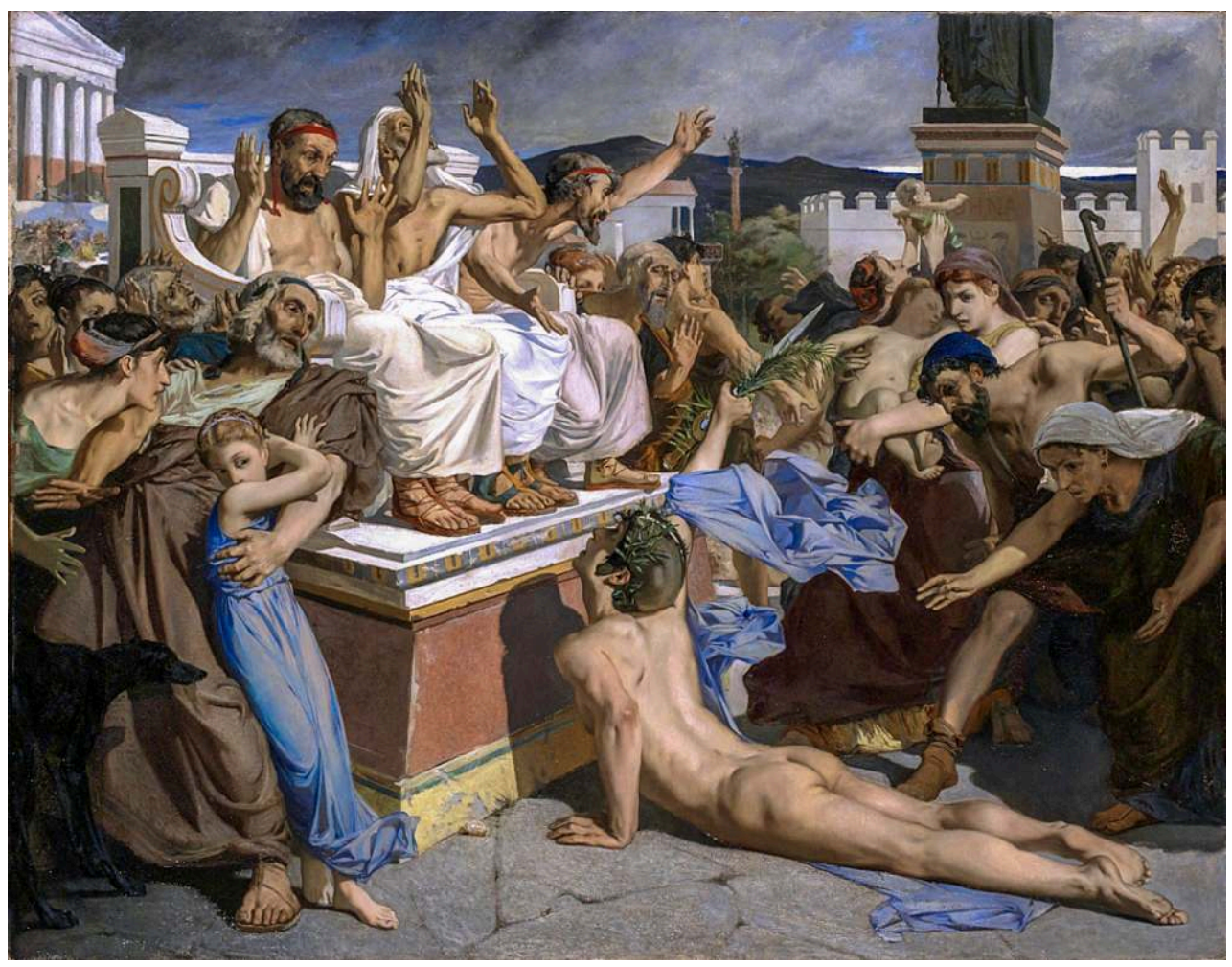

Le Soldat de Marathon, Luc Olivier Merson (1846-1920), huile sur toile (114 x $147 \mathrm{~cm})$ conservée à l'École nationale supérieure des beaux-arts (inv. PRP120), Paris. Prix de Rome de peinture en 1869. Reproduction RMN-Grand Palais.

En France, ces peintres exposent au Salon à partir de 1847, et sont qualifiés de « NéoGrecs ", « pompéistes » ou " Néo-Pompéiens » par Théophile Gautier. Nombre d'artistes se réunissent sous cette appellation ${ }^{50}$, qui offre l'originalité de transposer des situations modernes ou fantasmées dans un cadre antique qui se veut garant de perfection classique et de la tradition. Outre-Manche, c'est à partir de 1860 qu'apparaît le Classical Revival, qui se montre plus subtil dans son traitement du monde antique ${ }^{51}$ [fig. 13]. Frédéric Lord Leighton [fig. 14], peintre et sculpteur, est son représentant le plus connu et préside la Royal Academy pendant dix-huit ans, à partir de 1878. Il y laisse œuvres et dessins qui influencent nombre d'étudiants. Parmi les artistes britanniques formés par 
l'Académie, les plus épris de reconstitutions antiques, les peintres Lawrence AlmaTadema [fig. 15], Edward Poynter et Albert Moore.

Figure 14

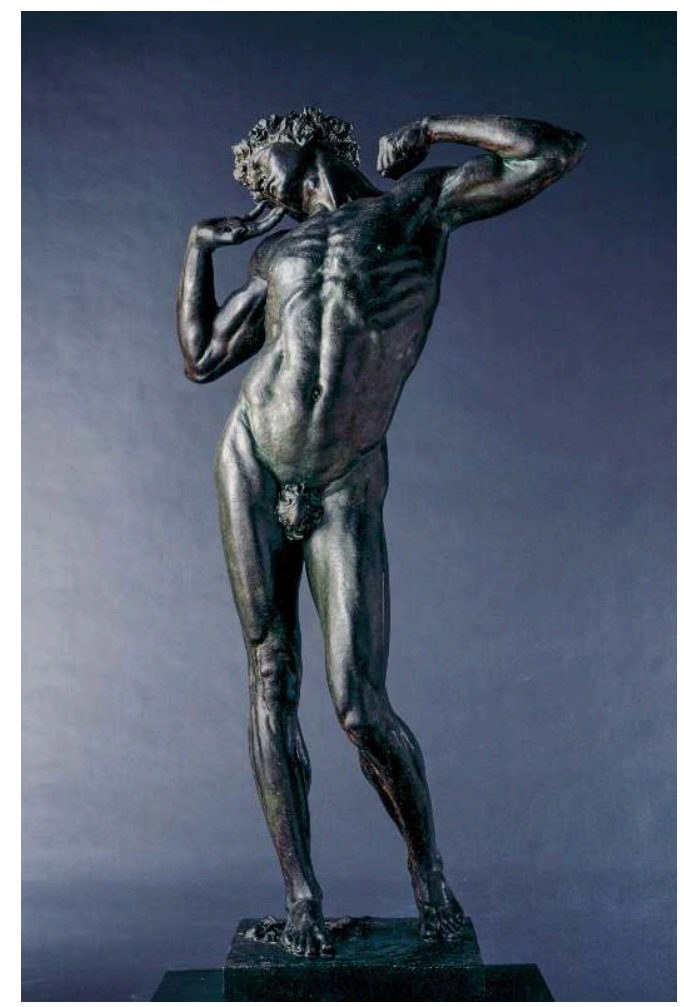

The Sluggard, Frédéric Lord Leighton, 1885, sculpture en plâtre $(190$ × 94 cm) conservée à la Royal Academy of Arts (inv. 03/1765), Londres.

(C) Paul Highnam (Royal Academy of Arts). 
Figure 15

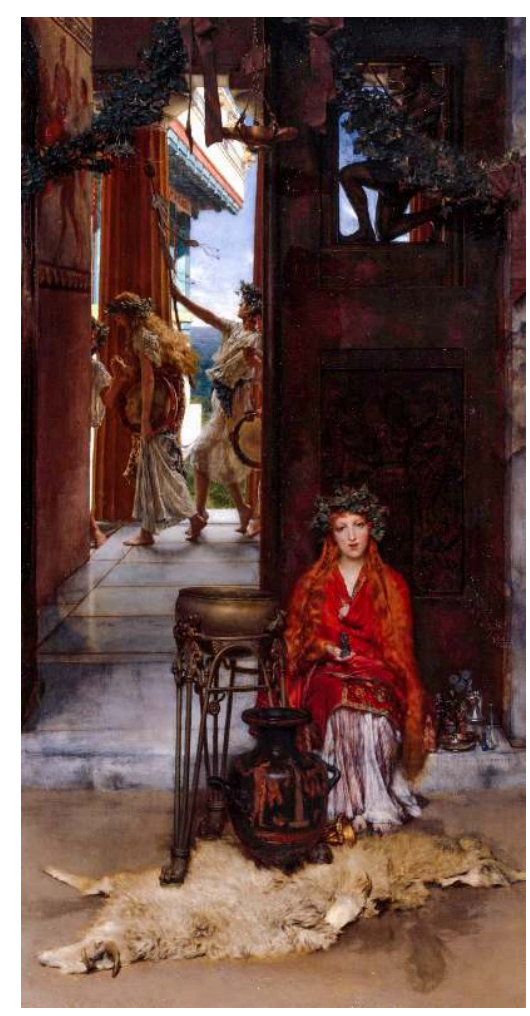

The Way to the temple, Sir Lawrence Alma-Tadema, 1882, huile sur toile $(101,5$ x $52 \mathrm{~cm})$ conservée à la Royal Academy of Arts (inv. 03/1021), Londres.

Reproduction Royal Academy of Art.

En Italie, dans les années 1860 , le «genre pompéien " est mis à l'honneur par des artistes formés à l'Académie des beaux-arts de Naples comme Domenico Morelli et Federico Maldarelli, dont les œuvres sont conservées au sein de l'Académie puis transférées dans les musées. En Allemagne, le groupe des Deutsch-Römer, actifs dès 1870 , revisitent à leur tour l'Antiquité dans des scènes de genre ou des reconstitutions des espaces gréco-romains.

Une fois estompée l'influence des écrits de Winckelmann, qui pouvait s'attarder sur les palpitations d'une narine ou la profondeur d'un nombril, les raisons didactiques persistent, car le plâtre permet d'apprécier plus facilement les volumes que le marbre. Les variations au sein des marbres peuvent en effet jouer sur la perception optique. Reste que la poésie des plâtres d'antiques, comme le montre le tableau de Johann Zoffany représentant la Salle des Antiques à New Somerset House, gît aussi dans leur capacité à s'animer à la lueur des lampes.

31 En dépit de la saturation atteinte par la prolifération de plâtres, notamment en Angleterre, dans les décors de particuliers ou les galeries privées ouvertes au public dans la seconde moitié du xixe siècle ${ }^{52}$, quelques mythes survivent, comme celui de la statue animée. C'est ce qui explique le succès de visites nocturnes des collections aux $\mathrm{XVIII}^{\mathrm{e}}$ et XIX ${ }^{\mathrm{e}}$ siècle, car " à cette lumière, le marbre coloré, rougi, devient chair [...]. Les détails anatomiques et les plus légères nuances du travail sont accusés par le reflet des flambeaux que les custodes approchent et promènent habilement le long des contours $^{53}$ ». C'est aussi ce qui explique qu'en dépit d'un usage didactique devenu 
largement obsolète aujourd'hui, ces collections continuent d'incarner la poésie intime de l'artiste en formation [fig. 16].

\section{Figure 16}

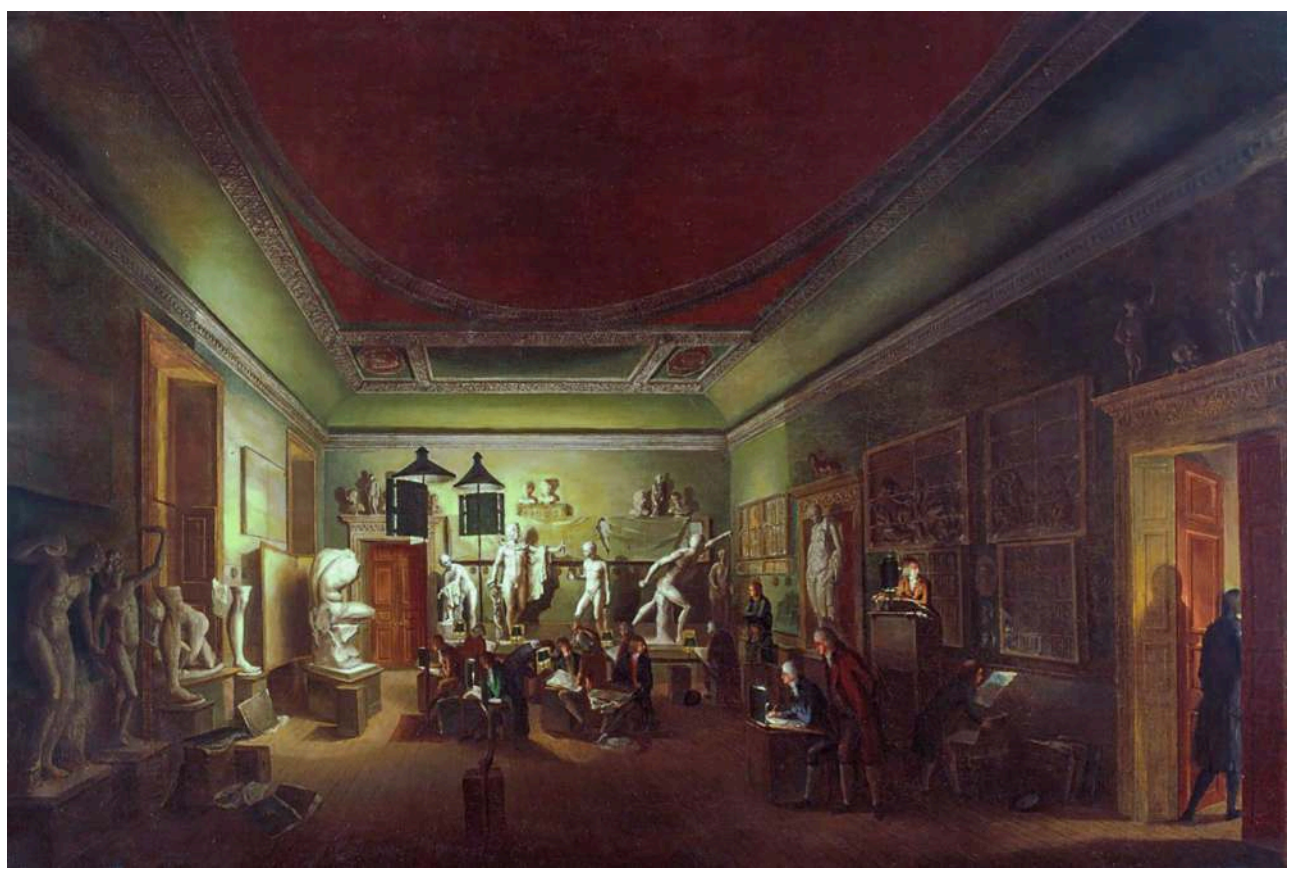

Salle des Antiques à New Somerset House, Johann Joseph Zoffany (1733-1810), 1780-1783, huile sur toile $(110,8 \times 164,1 \mathrm{~cm})$ conserve à la Royal Academy of Arts (inv. 03/846), Londres.

Reproduction Prudence Cuming Associates Limited (Royal Academy of Arts).

\section{NOTES}

1. GUICHARD Charlotte, «Les circulations artistiques en Europe, années 1680-années 1780 ", in BEAUREPAIRE Pierre-Yves \& POURCHASSE Pierrick (dir.), Les Circulations internationales en Europe. Années 1680 - années 1780, Rennes, Presses universitaires de Rennes, 2010, p. 385-398.

2. BÉDAT Claude, L'Académie des Beaux-Arts de Madrid:1744-1808. Contribution à l'étude des influences stylistiques et de la mentalité artistique de l'Espagne au XVIII ${ }^{\mathrm{e}}$ siècle, Toulouse, Association des publications de l'université de Toulouse-Le Mirail, 1973.

3. BAUDEZ Basile, «La Royal Academy of Arts au XVIII ${ }^{\mathrm{e}}$ siècle, une académie royale? ", Livraisons d'histoire de l'architecture, $\mathrm{n}^{\circ}$ 10, 2005, p. 123-136.

4. SAABYE Marianne, "The Royal Danish Academy of fine arts under the leadership of A. G. Moltke and J. F. Saly », Leids Kunsthistorisch Jaarboek, vol. 5-6, "Academies of art between Renaissance and Romanticism ", 1989, p. 527.

5. PEVSNER Nikolaus, Les Académies d'art, Paris, Gérard Monfort, 1999, p. 209.

6. ALTNER Manfred, Dresden: von der königlichen Kunstakademie zur Hochschule für Bildende Künste (1764-1989). Die Geschichte einer Institution, Dresde, Verlag der Kunst, 1990. 
7. HASKELL Francis \& PENNY Nicholas, Pour l'amour de l'Antique. La statuaire gréco-romaine et le goût européen, Paris, Hachette, 1999 [1981], p. 109.

8. RÖTTGEN Steffi, «Zum Antikenbesitz des Anton Raphael Mengs und zur Geschichte und Wirkung seiner Abguß- und Formensammlung », in BECK Herbert (dir.), Antikensammlungen im 18. Jahrhundert, Berlin, Mann, coll. « Frankfurter Forschungen zur Kunst », 1981, p. 129-148.

9. HASKELL Francis \& PENNY Nicholas, Pour l'amour de l'Antique..., p. 109.

10. S. N., Guide des étrangers à Vienne. Description de cette capitale de l'empire d'Autriche et des environs, Vienne, Mörschner et Jasper, 1838, p. 175.

11. HASKELL Francis \& PENNY Nicholas, Pour l'amour de l'Antique..., p. 117.

12. SIMON Robin \& STEVENS Mary Anne, The Royal Academy of Arts. History and collections, New Haven / Londres, Yale University Press, 2018, p. 450-453. Le carton a été mis en vente par l'Académie en 1962. La National Gallery s'en est portée acquéreur grâce à un mécénat public.

13. STEMMER Klaus, «Die Kunst hat nie ein Mensch allein besessen », Eine Ausstellungskatalog der Akademie der Künste und Hochschule der Künste, catalogue d'exposition (Akademie der Künste, Berlin, 9 juin-15 septembre 1996), Berlin, Henschel, 1996, p. 67-74.

14. JøRNÆS Bjarne, «Antiksalen på Charlottenborg », Meddelelser fra Thorvaldsens Museum, 1970, p. 48-65.

15. RÉAU Louis, «Les artistes russes à Paris au XVIII siècle ", Revue des études slaves, t. 3, 1923, p. 286-298.

16. Lettre du baron Grimm à Catherine II datée de 1782, citée dans RÉAU Louis, «Les artistes russes à Paris au XVIII ${ }^{\mathrm{e}}$ siècle ", p. 293.

17. DIDEROT Denis, Salon de 1765, Paris, Hermann, 1984, p. 144-146.

18. ROFFIDAL Émilie, «Les réseaux des académies et des écoles d'art en Europe méridionale (1740-1820) : projet et perspectives de recherche », Les Cahiers de Framespa. Nouveaux champs de l'histoire sociale, $\mathrm{n}^{\circ} 17$, «La prix de l'homme», 2014 [en ligne], https:// journals.openedition.org/framespa/3120?lang=en [lien valide en septembre 2020].

19. DELÉCLUZE Étienne-Jean, Louis David, son école et son temps. Souvenirs, Paris, Didier, 1863 [1855], p. 35-135.

20. OSTERKAMP Ernst, « Goethe et l'art français », Revue germanique internationale, $\mathrm{n}^{\circ} 12,1999$, p. 137-152.

21. MONTAIGLON Anatole de \& GUIFFREY Jules (dir.), Correspondance des directeurs de l'Académie de France à Rome avec les surintendants des Bâtiments, vol. 15, 1785-1790, Paris, Charasay frères, 1906, p. 372.

22. GUICHARD Charlotte, «Les circulations artistiques en Europe... ».

23. KIDERLEN Moritz \& KLUT Hans-Peter, Die Sammlung der Gipsabgüsse von Anton Raphael Mengs in Dresden. Katalog der Abgüsse, Rekonstruktionen, Nachbildungen und Modelle aus dem römischen Nachlass des Malers in der Skulpturensammlung, Staatliche Kunstsammlungen Dresden, Munich, Biering \& Brinkmann, 2006.

24. HERES Gerald, Dresdener Kunstsammlungen im 18. Jahrhundert, Leipzig, Seemann, 1991, p. 153-156.

25. RIONNET Florence, «Un instrument de propagande artistique: l'atelier de moulage du Louvre », Revue de l'art, nº 104, 1994, p. 49-50.

26. HASKELL Francis \& PENNY Nicholas, Pour l'amour de l'Antique..., p. 109.

27. REIMERS Henri de, L'Académie impériale des beaux-arts à St. Petersbourg depuis son origine jusqu'au règne d'Alexandre I' en 1807, Saint-Pétersbourg, Drechsler, 1807, p. 86.

28. DOTAL Christiane, «Paris-Rome-Madrid: l'influence de l'art français et romain sur les sculpteurs espagnols dans la première moitié du XIX siècle. Entre tradition et innovation ", Mélanges de la Casa de Velázquez, $n^{\circ}$ 36, vol. 2, « Le partage de l'eau (Espagne, Portugal, Maroc) », 2006, p. 255-279. Disponible en ligne, https://journals.openedition.org/mcv/2497 [lien valide en septembre 2020]. 
29. FREDERIKSEN Rune \& MARCHAND Eckart, Plaster casts. Making, collecting, and displaying from classical Antiquity to the present, Berlin / New York, De Gruyter, 2010, p. 227.

30. MICHAELIS Adolf, Der Parthenon, Leipzig, Breitkopf und Härtel, 1870, p. 84-87.

31. PINATEL Christiane, "La formation de la collection de moulages d'après l'antique à Versailles », Bulletin de la Société nationale des Antiquaires de France, 1999, p. 321.

32. BÉDAT Claude, L'Académie des Beaux-Arts de Madrid..., p. 186.

33. BÉDAT Claude, L'Académie des Beaux-Arts de Madrid..., p. 187.

34. MAIER ALLENDE Jorge, «II centenario de la Real Cédula de 1803. La Real Academia de la Historia y el inicio de la legislación sobre el Patrimonio Arqueológico y Monumental en España ", Boletín de la Real Academia de la Historia, $n^{\circ}$ 200, vol. 3, 2003, p. 439-473.

35. BÉDAT Claude, L'Académie des Beaux-Arts de Madrid..., p. 229.

36. DOTAL Christiane, « Paris-Rome-Madrid : l'influence de l'art français et romain... ».

37. CALVO MARTín Rocío, « Nuevos datos acerca de la colección Valparaíso de la Real Academia de Bellas Artes de San Fernando », Academia. Boletín de la Real Academia de Bellas Artes de San Fernando, n inconnu, 2014, p. 99-107.

38. À ce sujet, voir HOOCK Holger, The King's artists. The Royal Academy of Arts and the politics of British culture, 1760-1840, Oxford, Clarendon Press, 2003.

39. BARIDON Michel \& OGÉE Frédéric, «Art et nation en Grande-Bretagne : contexte et histoire d'un lien privilégié », Revue française de civilisation britannique, $\mathrm{n}^{\circ} 13$, vol. 4, « Art et nation en Grande-Bretagne au XVIII ${ }^{\mathrm{e}}$ siècle », 2006 [en ligne], https://journals.openedition.org/rfcb/1627 [lien valide en septembre 2020].

40. SIMON Robin \& STEVENS Mary Anne, The Royal Academy of Arts. History and collections, New Haven / Londres, Yale University Press, 2018, p. 435-436.

41. RÉAU Louis, L'Art russe, vol. 3, Le Classicisme, le romantisme, le $x^{e}$ siècle, Verviers, Gérard et Cie, 1968, p. 92.

42. KRIWDINA Olga A., "À propos des consignes données aux sculpteurs russes lauréats de l'académie à Rome ", Revue de l'art, 1994, vol. 104, 1994, p. 40-42. Disponible en ligne, https:// www.persee.fr/doc/rvart_0035-1326_1994_num_104_1_348125 [lien valide en septembre 2020].

43. MEDVEDKOVA Olga, "Diderot face à l'Académie des beaux-arts de Saint-Pétersbourg ", in S. N., La France et les Français à Saint-Pétersbourg, XVIII ${ }^{e}-X^{e}$ siècles, actes de colloque (SaintPétersbourg, 24-25 octobre 2003), Saint-Pétersbourg, Evropejskij Dom, 2005, p. 80-93.

44. DELACROIX Eugène, CEuvres littéraires, vol. 1, Études esthétiques, Paris, G. Crès, 1923, p. 107.

45. OSTERKAMP Ernst, « Goethe et l'art français ».

46. FRIEDRICH Caspar David, En contemplant une collection de peintures, Paris, José Corti, coll. « Domaine romantique », 2011, p. 126.

47. NERLICH France, «La peinture en Allemagne au $\mathrm{XIX}^{\mathrm{e}}$ siècle. Religion et politique: les Nazaréens et l'école de Düsseldorf ", Perspective, n² 2, 2008, p. 307-336.

48. GEOMINY Wilfred, Das Akademische Kunstmuseum der Universität Bonn unter der Direktion von Reinhard Kekulé, Amsterdam, Grüner, 1989, p. 47-60.

49. NEGRETE PLANO Almudena \& JORDAN DE URRIES Y DE LA COLINA Javier, Anton Raphael Mengs y la Antigüedad, catalogue d'exposition, Madrid, Real Academia de Bellas Artes de San Fernando, 2013, p. 64-79.

50. Notamment, pour ne citer que les plus célèbres : l'artiste suisse Charles Gleyre, Jean-Louis Hamon, Jean-Ernest Aubert, Auguste Toulmouche, Gustave-Rodolphe Boulanger, William Bouguereau, Alexandre Cabanel. Voir à ce sujet: COUËLLE Colombe, «Désirs d'Antique ou comment rêver le passé gréco-romain dans la peinture européenne de la seconde moitié du XIX ${ }^{\mathrm{e}}$ siècle ", Anabases, $\mathrm{n}^{\circ}$ 11, 2010, p. 21-54.

51. LIVERSIDGE Michael \& EDWARDS Catherine (dir.), Imagining Rome. British artists and Rome in the nineteenth century, Londres, Merell Holberton, 1996, p. 64.

52. HASKELL Francis \& PENNY Nicholas, Pour l'amour de l'Antique..., p. 159. 
53. VALÉRY Antoine-Claude, Voyages historiques, littéraires et artistiques en Italie. Guide raisonné et complet du voyageur et de l'artiste, Paris, Baudry / Aimé André, 1938.

\section{RÉSUMÉS}

Les principales académies européennes ont été fondées au long du XvIII ${ }^{\mathrm{e}}$ siècle, avec pour références principales les Académies de Paris et de Rome, tant sur le plan de leur fonctionnement que de leur méthode d'enseignement. À la faveur de la circulation intense des personnes et des œuvres à la fin du XVIII ${ }^{\mathrm{e}}$ siècle et dans la continuité de la culture humaniste de la Renaissance, la célébration européenne de l'antique s'épanouit au début du XIX siècle dans l'esthétique néoclassique, expliquant pour partie la présence massive de plâtres d'antiques dans les écoles d'art d'Europe. Cette présence est encore accentuée par l'immense impression produite auprès des artistes par les statues antiques rassemblées au musée Napoléon jusqu'en 1815. Les académies européennes veulent toutes réunir les chefs-d'œuvre de l'art antique sous la forme de collections de moulages.

Parallèlement à cette internationalisation des références plastiques se développent les premières réflexions autour d'une définition esthétique propre à chaque nation, que les guerres napoléoniennes ont contribué à nourrir. De nouveaux modèles apparaissent alors au sein des académies d'art, qu'il s'agisse de supports pédagogiques ou d'œuvres de réception. Le patrimoine des académies d'Europe se diversifie, sous l'impulsion de leurs directeurs respectifs. Les collections de plâtres d'antiques sont également enrichies de modèles nationaux, datant de l'époque médiévale ou renaissante. Ce patrimoine marque longtemps les méthodes d'apprentissage des artistes, parce qu'il propose une variété de solutions plastiques mais aussi parce qu'il incarne le rêve d'une histoire de l'art en trois dimensions, peuplée de statues animées à la lueur des lampes d'étude.

The main European academies we founded during the $18^{\text {th }}$ century, with the Paris and Rome academies as their main references, both for their ways of functioning and for their teaching method. Thanks to the important circulation of people and works of art in the late $18^{\text {th }}$ century, and as a continuation of the Renaissance era's humanist culture, Europe's celebration of Antiquity is blooming in the early $19^{\text {th }}$ century, with the neoclassic aesthetic, which partly explains the presence of so many plaster casts of antique works in European art schools. This presence is even more emphasized by the strong impressions made on artists by the antique statues that were gathered in the Napoleon Museum until 1815. European academies all want to gather the antique art masterpieces in the form of collections of plaster casts.

As a parallel to this internationalisation of plastic references, the first discussions on an aesthetical definition specific to each nation begin their development, fed by the Napoleonic Wars. New models start appearing in the art academies, either teaching supports or ordered works. European academies' patrimony becomes more diverse, led by their respective directors. The antique plaster casts collections are also enriched by national models dated from the Medieval or Renaissance eras. This patrimony has had a long-lasting impact on the teaching methods for artists, because it offers a variety of plastic solutions, and because it embodies the dream of a three-dimensional art history, filled with statues that come alive by the students' lamps. 


\section{INDEX}

Keywords : art teaching, art schools, art academies, pedagogy, museography, antique plaster casts collections, drawings collections, pedagogical collections, museum, Vienna Academy of Fine Arts, Madrid Academy of Fine Arts, San Fernando Royal Academy of Fine Arts, Royal Danish Academy of Fine Arts, Saint Petersburg Academy of Arts, Royal Academy of Art, Berlin Academy of Arts, Dresden Academy of Fine Arts

Mots-clés : enseignement artistique, écoles d'art, académie d'art, pédagogie, muséographie, collections de plâtres d'antiques, collection de dessin, collections pédagogiques, musée, Académie des beaux-arts de Vienne, Académie des beaux-arts de Madrid, Académie des beaux-arts de San Fernando, Académie des beaux-arts de Copenhague, Académie des beaux-arts de SaintPétersbourg, Académie des beaux-arts de Londres, Royal Academy of Art, Académie des beauxarts de Berlin, Académie des beaux-arts de Dresde

\section{AUTEUR}

\section{MORWENA JOLY-PARVEX}

Conservatrice au Centre des monuments nationaux, ministère de la Culture morwena.joly-parvex@monuments-nationaux.fr 